\title{
A New Analysis for Extending the Measurement Range of the Retardation-modulated Differential Interference Contrast (RM-DIC) Microscope
}

\author{
Hiroshi ISHIWATA, Masahide ITOH and Toyohiko YATAGAI \\ Institute of Applied Physics, University of Tsukuba
}

\begin{abstract}
While considering measurements of grating type samples with the retardation-modulated differential interference contrast (RM-DIC) microscope, we found a problem of phase objects. The problem is that smaller measured values than the actual phase distribution are obtained when phase objects are beyond the weak phase approximation range. We analyze the image characteristics of phase objects and show that the problem is caused by the effect of an image component which is proportional to the square of the phase distribution.

To overcome this disadvantage, we propose a new analysis method named self phase-correction (SPC). The SPC method corrects the phase distribution of the first order approximation under the weak phase condition and obtains the actual phase distribution of an object's phase beyond the weak phase approximation range. We propose a two-image SPC method and a three-image SPC method for the RM-DIC microscope, and examine the suitability of these methods.

We confirm that the three-image SPC method effectively overcomes the problem, and that the measurement range of the RM-DIC microscope can thus be extended to a half-wavelength phase difference.
\end{abstract}




\section{Introduction}

Differential interference contrast (DIC) microscopes ${ }^{[1,2]}$ are commonly used for observing the phase distribution of objects. The DIC microscope is a very powerful means of revealing detailed structures in living cells and small steps on the surfaces of semiconductor wafers. Existing DIC microscopes have high sensitivity and high horizontal resolution, but are unable to quantitatively measure the phase distribution of the phase object.

Previous researchers have tried to measure surface profiles and have discovered some methods of surface slope measurement with a DIC microscope ${ }^{[3,6-8,11]}$. Although these methods are useful for measuring surface slope, they cannot be extended to the reconstruction of the microstructures of an object because they use only the reflected light component to analyze the phase object. If the object has microstructures, the light used to illuminate it is diffracted by the edges of these surface structures, and the diffracted and reflected lights interfere to form an interference pattern. As a result, the phase information on edges that a DIC microscope is able to acquire from interference patterns, is limited to the average slope of the surface.

Previously, we developed the retardation-modulated DIC (RM-DIC) microscope which can quantitatively measure the phase distribution of a phase object ${ }^{[12]}$. We have been investigating quantitative measurements of phase objects by the RM-DIC microscope and found a problem that smaller measurement values than the real phase distribution of a phase object are obtained when the phase distribution has values larger than $\lambda / 4$ of the illuminating source wavelength.

In this paper, we describe what causes the problem and demonstrate a new analysis method of quantitative estimation from experimental results, and apply the new method to the RM-DIC microscope.

First, we outline the image characteristics of the DIC microscope and the principle of phase measurement by the RM-DIC microscope. Second, we describe the problem of measurement without the weak phase approximation range and discuss the cause of the problem. Third, we propose a new method of analyzing phase objects to extend the measurement range. Finally, we present the experimental results of 3-dimensional (3D) measurements made by the RM-DIC microscope and the new analysis method.

\section{Theory}

\subsection{The principle of the RM-DIC microscope}

According to the partial coherence theory, the image intensity distribution of a microscope is given by ${ }^{[4]}$

$I(x, y)=\iiint \int_{-\infty}^{\infty} R\left(f_{x}, f_{y}, f_{x}^{\prime}, f_{y}^{\prime}\right) O\left(f_{x}, f_{y}\right) O^{*}\left(f_{x}^{\prime}, f_{y}^{\prime}\right) \exp \left[-2 \pi i\left\{\left(f_{x}-f_{x}^{\prime}\right) x+\left(f_{y}-f_{y}^{\prime}\right) y\right\}\right] d f_{x} d f_{y} d f_{x}^{\prime} d f_{y}^{\prime},(1)$

where $R\left(f_{x}, f_{y}, f_{x}^{\prime}, f_{y}^{\prime}\right)$ denotes the transmission cross-coefficient (TCC) and is expressed as

$$
R\left(f_{x}, f_{y}, f_{x}^{\prime}, f_{y}^{\prime}\right)=\iint_{-\infty}^{\infty} Q(\xi, \varsigma) p\left(\xi+f_{x}, \varsigma+f_{y}\right) p^{*}\left(\xi+f_{x}^{\prime}, \varsigma+f_{y}^{\prime}\right) d \xi d \varsigma .
$$

In Eqs. (1) and (2), $O\left(f_{x}, f_{y}\right)$ is the Fourier transform of an object $o(x, y), p(\xi, \varsigma)$ is the pupil function of an imaging system and $Q(\xi, \varsigma)$ is the intensity distribution at the pupil of the illuminating optical system.

To simplify the description, we use a simple model that has a one-dimensional phase distribution along the $\mathrm{x}$-axis and which is constant along the y-axis. We discuss the intensity distribution of a microscope image in the case of a pure phase object. We assume the phase distribution of the object to be the function $\phi(x)$ with uniform reflectivity $C$. According to the weak phase approximation, $o(x, y)$ and $O\left(f_{x}, f_{y}\right)$ are expressed as 


$$
o(x)=C \exp \{i \phi(x)\}=C\left\{1+i \phi(x)-\frac{1}{2} \phi(x)^{2}\right\}
$$

and

$$
O\left(f_{x}\right)=C\left\{\delta\left(f_{x}\right)+i \Phi\left(f_{x}\right)-\frac{1}{2} \Phi\left(f_{x}\right) \otimes \Phi^{*}\left(f_{x}\right)\right\}
$$

respectively, where $\Phi\left(f_{x}\right)$ is the Fourier transform of the phase distribution of $\phi(x)$. The symbol $\otimes$ is the convolution operator.

Substituting Eq. (4) and TCC of the DIC microscope (Eq. (2)) into Eq. (1), the image intensity distribution of the DIC microscope is written as ${ }^{[12]}$

$$
\begin{aligned}
& I(x, \theta)=C {\left[\frac{1}{2}(1-\cos \theta)\left\{M(0)-\int_{-\infty}^{\infty} \cos (\pi \Delta f) M\left(f_{x}\right) \Phi\left(f_{x}\right) \otimes \Phi^{*}\left(f_{x}\right) \exp \left(-2 \pi i f_{x} x\right) d f_{x}\right\}\right.} \\
&+ i \sin \theta \int_{-\infty}^{\infty} \sin \left(\pi \Delta f_{x}\right) M\left(f_{x}\right) \Phi\left(f_{x}\right) \exp \left(-2 \pi i f_{x} x\right) d f_{x} \\
&+\frac{1}{2} \int_{-\infty}^{\infty}\left\{1-\cos \left(2 \pi \Delta f_{x}+\theta\right)\right\} m_{0}\left(f_{x}\right) \Phi\left(f_{x}\right) \Phi^{*}\left(f_{x}\right) d f_{x} \\
&\left.+\frac{1}{2} \int_{-\infty}^{\infty}\left\{\cos \theta+\cos \left(2 \pi \Delta f_{x}\right)\right\} m_{d}\left(f_{x}\right) \Phi\left(f_{x}\right) \Phi^{*}\left(-f_{x}\right) \exp \left(-4 \pi i f_{x} x\right) d f_{x}\right] \\
& M(0)=\iint_{-\infty}^{\infty} Q(\xi, \varsigma) p(\xi, \varsigma) p^{*}(\xi, \varsigma) d \xi d \varsigma, \quad M\left(f_{x}\right)=\iint_{-\infty}^{\infty} Q(\xi, \varsigma) p\left(\xi+f_{x}, \varsigma\right) p^{*}(\xi, \varsigma) d \xi d \varsigma, \\
& m_{0}\left(f_{x}\right)=\iint_{-\infty}^{\infty} Q(\xi, \varsigma) p\left(\xi+f_{x}, \varsigma\right) p^{*}\left(\xi+f_{x}, \varsigma\right) d \xi d \varsigma, \quad m_{d}\left(f_{x}\right)=\iint_{-\infty}^{\infty} Q(\xi, \varsigma) p\left(\xi+f_{x}, \varsigma\right) p^{*}\left(\xi-f_{x}, \varsigma\right) d \xi d \varsigma,
\end{aligned}
$$

where $\Delta$ is the shear amount of DIC images, and $\theta$ is the retardation between two sheared images in the DIC microscope. It is assumed that microstructures are on a flat plane and the phase distribution of the microstructures is small. In this case we can neglect the effects of the third and fourth terms of Eq. (5), which allows us to approximate Eq. (5) as

$$
I(x, \theta)=C\left\{\frac{1}{2}(1-\cos \theta) M(0)+i \sin \theta \int_{-\infty}^{\infty} \sin \left(\pi \Delta f_{x}\right) M\left(f_{x}\right) \Phi\left(f_{x}\right) \exp \left(-2 \pi i f_{x} x\right) d f_{x}\right\}
$$

As the image contrast changes as a function of $\theta$, we take two DIC images with different retardations $( \pm \theta)$ and subtract these two DIC images to obtain the difference between them:

$$
I(x, \theta)-I(x,-\theta)=2 i C \sin \theta \int_{-\infty}^{\infty} \sin \left(\pi \Delta f_{x}\right) M\left(f_{x}\right) \Phi\left(f_{x}\right) \exp \left(-2 \pi i f_{x} x\right) d f_{x} .
$$

Equation (7) shows the phase component of the DIC image. We can therefore extract the phase component from the DIC image by subtracting two DIC images with different retardations $( \pm \theta)$.

Since Eq. (7) includes the incident light parameter and absorption constant $C$ (the amplitude component), to extract the phase component we need to normalize Eq. (7), and so we sum the two DIC images:

$$
I(x, \theta)+I(x,-\theta)=C(1-\cos \theta) M(0) .
$$

Equation (8) shows the intensity of the incident light on an object from the illuminating optics at retardation $\theta$.

Both the phase and the amplitude components can be extracted from two DIC images with different retardations $( \pm \theta$ ).

From Eqs. (7) and (8),

$$
\begin{aligned}
\frac{I(x, \theta)-I(x,-\theta)}{I(x, \theta)+I(x,-\theta)} & =\frac{2 i \sin \theta}{(1-\cos \theta) M(0)} \int_{-\infty}^{\infty} \sin \left(\pi \Delta f_{x}\right) M\left(f_{x}\right) \Phi\left(f_{x}\right) \exp \left(-2 \pi i f_{x} x\right) d f_{x} \\
& =\frac{2 i \sin \theta}{(1-\cos \theta)} \int_{-\infty}^{\infty} M T F_{D}\left(f_{x}\right) \Phi\left(f_{x}\right) \exp \left(-2 \pi i f_{x} x\right) d f_{x} \\
& =\frac{2 \sin \theta}{(1-\cos \theta)} \phi(x) \otimes L S F_{D}(\Delta)
\end{aligned}
$$


where $\quad \operatorname{MTF}_{D}\left(f_{x}\right)=\frac{\sin \left(\pi \Delta f_{x}\right) M\left(f_{x}\right)}{M(0)}, \quad L S F_{D}(\Delta)=\int_{-\infty}^{\infty} M T F_{D}\left(f_{x}\right) \exp \left(-2 \pi i f_{x} x\right) d f_{x}$,

$M_{D T}(f)$ is the MTF of the DIC microscope, and $L S F_{D}(\Delta)$ is the line spread function of the DIC microscope with the amount of shear $\Delta$.

Equation (9) shows that the normalized phase component of the DIC image represents the convoluted phase distribution of the object observed by the MTF of the DIC microscope given by

$$
\phi(x)=\frac{1-\cos \theta}{2 \sin \theta} \cdot \operatorname{Decon}\left[\frac{I(x, \theta)-I(x,-\theta)}{I(x, \theta)+I(x,-\theta)}\right],
$$

where $\operatorname{Decon}[\cdot]$ is the deconvolution function and is expressed as

$$
\text { Decon }[g(x)]=\cdot \mathfrak{I}^{-1}\left[\mathfrak{I}\{g(x)\} / \text { iMTF }_{D}(f)\right]
$$

where $g(x)$ is any function with Fourier transform, $\mathfrak{I}()$ is the Fourier transform function and $\mathfrak{I}^{-1}()$ is the inverse Fourier transform function.

From Eq. (10) we can precisely measure the phase distribution of the observed object as we deconvolute the phase component of the DIC image using the MTF of the DIC microscope with the amount of shear $\Delta$.

By taking two DIC images with different retardations $( \pm \theta)$ and analyzing them, we can obtain the precise phase distribution of the object ${ }^{[12]}$.

\subsection{A problem of the weak phase approximation and self phase-correction method}

In the weak phase approximation we can neglect the image component proportional to the square of the phase distribution in Eq. (5). However, beyond the weak phase approximation range, the image component proportional to the square of the phase distribution in Eq. (5) cannot be eliminated and an effect of it appears.

We describe this effect and introduce a new analysis method to resolve the problem.

\section{- A problem of the weak phase approximation}

Generally, light going out from a phase object has the zeroth order light component $A_{C}$ transmitted or reflected from the object and the first order diffracted light $A_{S}$. These components are represented by the vectors shown in Fig. 1.

The complex amplitude $\mathrm{A}$ of the light going out from a phase object is given by

$$
A=A_{0} \exp (i \varphi)=A_{0} \cos \phi+i A_{0} \sin \phi=A_{C}+i A_{S}
$$

where $A_{0}$ is the amplitude of the incident light.

The image intensity distribution of the DIC microscope $I$ is written as

$$
I=\left|b A_{C}+i A_{S}\right|^{2}
$$

where $b$ is a parameter to adjust the phase difference between $A_{C}$ and $A_{S}$.

In the DIC microscope, the parameter $b$ becomes an imaginary number as the retardation is adjusted, so the image intensity distribution can be rewritten as

$$
I=A_{C}{ }^{2}+A_{S}{ }^{2}+2 A_{C} \cdot A_{S} .
$$

Equation (11) indicates that an image of the DIC microscope consists of three image components. One of them is the zeroth order light image as a background, another is the first order diffracted light image proportional to the square of the phase distribution, and the last one is the interference image between the zeroth order and the first order diffracted light.

A DIC image contains interference between the light including the phase component $A_{S}$ and the transmitted or reflected light component $A_{C}$. 
The image component $I_{S}$ proportional to the phase distribution of the DIC microscope is written as

$$
I_{S}=A_{S} \cdot A_{C}=A_{0}^{2} \sin \phi \cdot \cos \phi .
$$

Equation (12) is proper for the deconvoluted result of the second term of Eq. (5).

In the same way as Eq. (12), the zeroth order light image component $I_{C}$ and the image component proportional to the square of the phase distribution $I_{d}$ are each given by

$$
\begin{gathered}
I_{C}=A_{C} \cdot A_{C}=A_{0}{ }^{2} \cos ^{2} \phi, \\
I_{d}=A_{S} \cdot A_{S}=A_{0}{ }^{2} \sin ^{2} \phi .
\end{gathered}
$$

Each of Eqs. (13) and (14) is related with the deconvoluted results of the first term and the fourth term of Eq. (5).

When the phase distribution is in the weak phase approximation range, it seems small and the image component $I_{d}$ is considered to be zero.

The image intensity distribution of the DIC microscope $I$ can be rewritten as

$$
I=I_{C}+I_{S}
$$

From Eqs. (12) and (13) the phase distribution is written as

$$
\phi=\tan ^{-1}\left(I_{S} / I_{C}\right) \text {. }
$$

In order to measure the phase distribution, each of image components $I_{C}$ and $I_{S}$ is extracted from the image intensity distribution $I$ and then the phase distribution can be calculated by Eq. (16).

However, when the phase distribution goes outside of the weak phase approximation range, the image component $I_{d}$ cannot be considered to be zero.

As the image components $I_{C}$ and $I_{d}$ cannot be separately extracted from a DIC image, the measurement result $\phi_{w}$ assuming the weak phase approximation is given by

$$
\phi_{w}=\tan ^{-1}\left\{I_{S} /\left(I_{C}+I_{d}\right)\right\} \text {. }
$$

Then the phase distribution $\phi_{w}$ is smaller than the actual value $\phi$.

From Eqs. (13) and (14), the expression inside the brackets \{\} of Eq. (17) can be replaced by

$$
\frac{I_{S}}{I_{C}+I_{S}}=\frac{I_{S}}{I_{0}}=\sin \phi \cos \phi=\frac{\sin 2 \phi}{2} .
$$

When the phase value $\phi$ exceeds a quarter-wavelength, Eq. (18) shows that the measurement result $\phi_{w}$ decreases as the actual phase $\phi$ increases, and that the difference between the measurement and actual phase value widens.

Finally, the DIC image is not formed and the phase distribution cannot be measured when the phase value reaches half the wavelength of the illumination light.

In order to get the actual phase distribution of the phase object, we need to extract the image components $I_{C}$ formed by the transmitted or reflected light from the DIC image.

\section{- Self phase-correction method}

Let us describe a new method to extract the image components $I_{C}$ from the DIC image of the phase object beyond the weak phase approximation range.

In this method, we predict the image component $I_{d}$ with the phase distribution assumed in the weak phase approximation, and then correct the measurement value.

As shown in Fig. 2, from Eqs. (13) and (14) the relationship between $I_{0}, I_{S}$ and $I_{C}$ can be considered to be 
$I_{C}=\sqrt{I_{0}^{2}-I_{S}^{2}}$. Therefore, $I_{C}$ can be approximately rewritten as $I_{C}=I_{0}\left(1-I_{S}^{2} / 2 I_{0}^{2}\right)$, so $I_{S} / I_{C}$ is replaced by

$$
\frac{I_{S}}{I_{C}}=\frac{I_{S}}{\sqrt{I_{0}^{2}-I_{S}^{2}}} \cong \frac{I_{S}}{I_{0}\left(1-I_{S}^{2} / 2 I_{0}^{2}\right)}=\frac{I_{S} / I_{0}}{\left(1-I_{S}^{2} / 2 I_{0}^{2}\right)} .
$$

We set $\phi_{w}$ to the phase value obtained by the first order approximation by assuming the weak phase approximation.

$$
\phi_{w}=I_{S} / I_{0}
$$

Substituting Eqs. (19) and (20) into Eq. (16), the actual phase value $\varphi$ beyond the weak phase approximation range is given by

$$
\varphi=\tan ^{-1}\left[\phi_{w} /\left(1-\phi_{w}^{2} / 2\right)\right] .
$$

In this method, the image component proportional to the square of the phase distribution is approximately required by the phase distribution $\phi_{w}$ obtained by assuming the weak phase approximation, and its effect is corrected by the phase distribution $\phi_{w}$.

We can measure the phase distribution beyond the weak phase approximation range by Eqs. (20) and (21) in the same way as the weak phase approximation.

In this paper, we name this new analysis method "self phase-correction (SPC)" because it corrects the result by the measured phase distribution of the first order approximation assuming the weak phase approximation.

\subsection{Methods for extending the measurement range of the RM-DIC microscope}

This section explains how to apply the SPC method to the RM-DIC microscope and how to extend the measurement range of the RM-DIC microscope.

\section{- Self phase-correction method with two images}

First we introduce the SPC method with two DIC images. We take two DIC images with different retardations $( \pm \theta)$ and make the subtracted and summed images from these two images.

When a phase object is beyond the weak phase approximation, from Eq. (8) the image component $I_{S}$ is written as

$$
I_{S}=I(x, \theta)-I(x,-\theta) \text {. }
$$

The summed image is given by

$$
\begin{aligned}
& I(x, \theta)+I(x,-\theta) \\
&=C\left[(1-\cos \theta)\left\{M(0)-\int_{-\infty}^{\infty} M\left(f_{x}\right) \Phi\left(f_{x}\right) \otimes \Phi^{*}\left(f_{x}\right) \exp \left(-2 \pi i f_{x} x\right) d f_{x}\right\}\right. \\
&\left.+\int_{-\infty}^{\infty}\{\cos \theta+1\} m_{d}\left(f_{x}\right) \Phi\left(f_{x}\right) \Phi^{*}\left(-f_{x}\right) \exp \left(-4 \pi i f_{x} x\right) d f_{x}\right] .
\end{aligned}
$$

Equation (23) shows that the image component $I_{d}$ proportional to the square of the phase distribution appears in the summed image, unlike the weak phase approximation.

Noting the spatial frequency, the image component $I_{C}$ can be approximately separated by spatial frequency filtering because the image component $I_{d}$ shown in second term of Eq. (23) has of twice frequency of the phase object.

The image component $I_{C}$ can be extracted by low-pass filtering the summed image:

$$
I_{C}=\operatorname{Low}[I(x, \theta)+I(x,-\theta)]
$$

where Low $[\cdot]$ is the low-pass operation passing less than a half of the cut-off frequency.

Deconvoluting the result of Eq. (22) divided by Eq. (24) in the same way as Eq. (10), the phase distribution of the first order approximation $\phi_{w}$ can be derived as 


$$
\phi_{w}(x)=\frac{1-\cos \theta}{2 \sin \theta}\left\{\operatorname{Decon}\left[\frac{I(x, \theta)-I(x,-\theta)}{\operatorname{Low}[I(x, \theta)+I(x,-\theta)]}\right]\right\} .
$$

Then applying the phase distribution $\phi_{w}$ to the SPC method, the corrected phase is obtained as

$$
\phi=\tan ^{-1}\left[\phi_{w} /\left(1-\phi_{w}^{2} / 2\right)\right] .
$$

In this paper, we name this new analysis method of applying the SPC method to the temporary phase distribution with the two DIC images as "two-image SPC method".

\section{- Self phase-correction method with three images}

By low-pass filtering the two DIC images with different retardations $( \pm \theta)$ shown in Eq. (24), the two-image SPC method separates the image component $I_{d}$ from the summed image by the difference of spatial frequency.

In order to extract the objects' phase correctly, we propose another method using three DIC images with different retardations ( $\pm \theta$ and 0$)$.

First, we describe how to reduce the effect of the image component $I_{d}$ by three DIC images with different retardations $( \pm \theta$ and 0$)$.

The amount of shear is assumed to be very small, so the image intensity distribution of a DIC image is approximately given by

$$
\begin{aligned}
I(x, \theta)=C & {\left[\frac{1}{2}(1-\cos \theta)\left\{M(0)-\int_{-\infty}^{\infty} M\left(f_{x}\right) \Phi\left(f_{x}\right) \otimes \Phi^{*}\left(f_{x}\right) \exp \left(-2 \pi i f_{x} x\right) d f_{x}\right\}\right.} \\
& +i \sin \theta \int_{-\infty}^{\infty} \sin \left(\pi \Delta f_{x}\right) M\left(f_{x}\right) \Phi\left(f_{x}\right) \exp \left(-2 \pi i f_{x} x\right) d f_{x} \\
& \left.+\frac{1}{2} \int_{-\infty}^{\infty}\{\cos \theta+1\} m_{d}\left(f_{x}\right) \Phi\left(f_{x}\right) \Phi^{*}\left(-f_{x}\right) \exp \left(-4 \pi i f_{x} x\right) d f_{x}\right] .
\end{aligned}
$$

When the retardation is zero, the image intensity distribution is approximately written as

$$
I(x, 0)=\int_{-\infty}^{\infty} m_{d}\left(f_{x}\right) \Phi\left(f_{x}\right) \Phi^{*}\left(-f_{x}\right) \exp \left(-4 \pi i f_{x} x\right) d f_{x} .
$$

From Eq. (27) the summed image of different retardations $( \pm \theta)$ is given by

$$
\begin{aligned}
I(x, \theta)+ & I(x,-\theta) \\
= & C\left[(1-\cos \theta)\left\{M(0)-\int_{-\infty}^{\infty} M\left(f_{x}\right) \Phi\left(f_{x}\right) \otimes \Phi^{*}\left(f_{x}\right) \exp \left(-2 \pi i f_{x} x\right) d f_{x}\right\}\right. \\
& \left.+\int_{-\infty}^{\infty}\{\cos \theta+1\} m_{d}\left(f_{x}\right) \Phi\left(f_{x}\right) \Phi^{*}\left(-f_{x}\right) \exp \left(-4 \pi i f_{x} x\right) d f_{x}\right] .
\end{aligned}
$$

Equation (29) can be rewritten using Eq. (28) as

$$
\begin{aligned}
I(x, \theta)+I & (x,-\theta)-(\cos \theta+1) \cdot I(x, 0) \\
& =C(1-\cos \theta)\left\{M(0)-\int_{-\infty}^{\infty} M\left(f_{x}\right) \Phi\left(f_{x}\right) \otimes \Phi^{*}\left(f_{x}\right) \exp \left(-2 \pi i f_{x} x\right) d f_{x}\right\} .
\end{aligned}
$$

Equation (30) shows that the image component $I_{C}$ can be extracted from the summed image by calculating the three images with different retardations ( $\pm \theta$ and 0 ). Therefore, the phase distribution of the object is given by

$$
\phi(x)=\frac{1-\cos \theta}{2 \sin \theta} \cdot \tan ^{-1}\left\{\operatorname{Decon}\left[\frac{I(x, \theta)-I(x,-\theta)}{I(x, \theta)+I(x,-\theta)-(\cos \theta+1) \cdot I(x, 0)}\right]\right\} .
$$

We call this method using three DIC images with different retardations ( $\pm \theta$ and 0 ) given by Eq. (30) the "retardation zero image correction (RZC) method".

Next, we introduce an improvement of the RZC method. With the RZC method we can approximate Eq. (27) by assuming that the amount of shear in a DIC microscope is very small. 
As some approximation error remains in Eq. (27), we consider a new method that combines the SPC method and RZC method to reduce this approximation error.

We take three DIC images with different retardations ( $\pm \theta$ and 0$)$ and calculate the phase value by the RZC method given by Eq. (31).

The calculated phase value is set to the phase distribution of the first order approximation $\phi_{w}$ as

$$
\phi_{w}(x)=\frac{1-\cos \theta}{2 \sin \theta} \tan ^{-1}\left\{\operatorname{Decon}\left[\frac{I(x, \theta)-I(x,-\theta)}{I(x, \theta)+I(x,-\theta)-(\cos \theta+1) \cdot I(x, 0)}\right]\right\} .
$$

To reduce the effect of the image component $I_{d}$ we apply the temporary phase distribution $\phi_{w}$ to the SPC method. The measurement result $\phi$ is given by

$$
\phi=\tan ^{-1}\left[\phi_{w} /\left(1-\cdot \phi_{w}^{2} / 2\right)\right] .
$$

In this paper we call this combination of the RZC method and SPC method the "three-image SPC method".

The RZC method and the three-image SPC method proposed here yield a result that is closer to the true phase distribution of the observed object than the two-image SPC method because these methods obtain the first order approximation $\phi_{w}$ by the retardation 0 image which approximately consists of only image component $I_{d}$.

\section{Experiments}

\section{Experimental set-up}

We have developed a new DIC microscope that includes a modulating retardation unit in the illuminating optics of a conventional Olympus BX51 microscope, and have applied our new analysis method to this microscope. We call the new DIC microscope the RM-DIC microscope. Figure 3 shows a block diagram of our setup.

The measuring system has four objective lenses $(10 \times, 20 \times, 50 \times, 100 \times)$. We can choose the magnification by changing the objective lens. Using a halogen lamp, we can select the observation wavelength by adjusting the interference filter. We can control the retardation of the DIC microscope with the phase modulator. The phase modulator is controlled to within $\pm 1^{\circ}$ accuracy by a desktop computer. The CCD camera is a $1 / 3$ " (B/W) VGA type and is connected to a frame grabber in the desktop computer.

\section{Samples}

The samples were silicon wafers with grating patterns formed by a semiconductor process, as illustrated in Fig. 4. The grating pattern had different line widths $L$ ranging from $0.5 \mu \mathrm{m}$ to $10 \mu \mathrm{m}$ and was covered with a thin aluminum layer in the same way as the phase objects. The depth $d$ was maintained at $20 \mathrm{~nm}, 50 \mathrm{~nm} 75 \mathrm{~nm}$ and $90 \mathrm{~nm}$ by the thickness of the $\mathrm{SiO}_{2}$ patterns.

\section{Results and discussion}

In this experiment we assumed that the microscope had no aberration. We used an objective lens having a magnification of larger than 50 because the cut-off frequency of the imaging optics was kept lower than the Nyquist frequency to decrease sampling errors in the CCD camera. The illuminating wavelength was determined as $550 \pm 30 \mathrm{~nm}$ by an interference filter (IF550 produced by OLYMPUS). Taking three DIC images, we set the three different retardations of $\pm \pi / 2$ and 0 .

When using the two-image SPC method we analyzed two DIC images with two different retardations of $\pm \pi / 2$. When using the three-image SPC method we used all three DIC images.

For the depth measurement we used four kinds of grating-type samples with $d=20 \mathrm{~nm}, \mathrm{~d}=50 \mathrm{~nm}, \mathrm{~d}=75 \mathrm{~nm}$ and $\mathrm{d}=$ 
$90 \mathrm{~nm}$.

Our RM-DIC microscope can take independent measurements of the line width of samples because it measures them using the deconvolution method.

According to the imaging area of the CCD camera in the observation by the $50 \times$ objective lens, we chose three measuring regions (a), (b) and (c) with different line widths in each sample shown in Fig. 5. In region (a) the grating samples had line widths of 1.0, 1.2 and $1.4 \mu \mathrm{m}$, in region (b) 1.6 and $1.8 \mu \mathrm{m}$, and in region (c) 2.0 and $3.0 \mu \mathrm{m}$.

\subsection{A problem of the weak phase approximation}

First, in order to explain the problem of the weak phase approximation, the measurement results are shown in Table 1 and Fig. 6 for the $50 \times$ objective lens and analyzed by Eq. (10).

Table 1 and Fig. 6 show the relation between the depth of the sample and the measurement result for each line width, revealing the problem of the measurement result being smaller than the original depth of samples beyond $50 \mathrm{~nm}$.

Here, for example, we show the result for the $75 \mathrm{~nm}$ depth sample in Fig. 7 with the cross-section of each part.

Figure 6 confirms that the difference between the actual depth and the measurement value assuming the weak phase approximation increases as the depth $d$ exceeds $50 \mathrm{~nm}$ in all line-width parts.

The results shown in Fig. 6 represent the effect of the image component proportional to the square of the phase distribution described in the previous chapter.

Therefore, assuming the weak phase approximation, measurement errors increase beyond $50 \mathrm{~nm}$ depth. Additionally, Eq. (18) and Fig. 6 reveal that the measurement error is extended to beyond $75 \mathrm{~nm}$ depth which has over one-quarter wavelength of the illuminating light on optical pass difference.

\subsection{Results by the SPC method}

Experimental results using our new analysis methods described in the previous section are given below. We used the same DIC images of the results shown in Table 1 and Fig. 6 for reconstructing the phase distribution.

\section{- Results by the two-image SPC method}

First, we present measurement results by the two-image SPC method. We analyze the two DIC images with different retardations of $\pm \pi / 2$ by the two-image SPC method.

We make a subtracted image and a summed image from the DIC images with different retardations $( \pm \pi / 2)$ and obtain the phase distribution $\phi_{w}$ from Eq. (25) with the subtracted image and the summed image using a small-area averaging filter as the low-pass filter.

Then we convert the phase distribution $\phi_{w}$ to the corrected value by using the two-image SPC method. The result is shown in Table 2 versus line width and in Fig. 8 to compare with the weak phase approximation.

The results show that the two-image SPC method effectively corrects the phase distribution independently of the line width of the sample and that it can extend the measurement range of the RM-DIC microscope beyond $50 \mathrm{~nm}$ depth.

\section{- Results by the three-image SPC method}

Next, we present results using three DIC images with different retardations of $\pm \pi / 2$ and 0 by the three-image SPC method. We convert the phase distribution $\phi_{w}$ obtained by the RZC method to the measurement value with the three-image SPC method and show the results in Table 4. The results measured by the RZC method are given as a 
reference in Table 3.

In order to compare the weak phase approximation with the two-image SPC method, the RZC method and the three-image SPC method, the measurement results for each method are given in Fig. 8.

Figure 8 shows that the RZC method and the three-image SPC method correct the phase distribution better than the two-image SPC method. Especially, the values measured by the three-image SPC method are closer to the true phase distribution of the objects.

These methods give a wider measurement range of the RM-DIC microscope than the two-image method. Although the two-image SPC method extends the measurement range beyond $50 \mathrm{~nm}$ depth, it is difficult to correct the effect up to a 75 nm depth sample.

The RZC method with three DIC images can approximately reduce the effect of the image component $I_{d}$ and extend the measurement range up to a $75 \mathrm{~nm}$ depth sample.

The three-image SPC method has very good correction performance and can measure sample depths up to $90 \mathrm{~nm}$. This would extend the measurement range to a half-wavelength because it corrects the phase distribution of the first order approximation $\phi_{w}$ obtained by the RZC method to the value measured by the SPC method.

Thus, the three-image SPC method can reduce measurement errors based on the image component proportional to the square of the phase distribution, obtain the correct depth value, and extend the measurement range of the RM-DIC microscope.

\section{Conclusion}

When phase objects beyond the weak phase approximation range are measured, the method based on the weak phase approximation gives smaller results than the original phase distribution, for the binary gratings considered here. This measurement error is thought to be caused by the image component proportional to the square of the phase distribution interfering with the measurement result.

In order to reduce the measurement error, we proposed the SPC method that converts the phase distribution of the first order approximation obtained by assuming the weak phase approximation to the measurement value.

We presented application of the two-image SPC method and three-image SPC method to our RM-DIC microscope and examined the performance of each method. We confirmed that the three-image SPC method reduces the error for groove depths of up to $90 \mathrm{~nm}$.

We showed that the three-image SPC method is a very useful tool to extend the measurement range up to a half-wavelength phase difference by the RM-DIC microscope without dependence on the line width of samples. However, a drawback of a contour inversion taken from a DIC image of an object beyond the half-wavelength remains as contrasts of DIC images of beyond half-wavelength phase objects have inverted distribution to under half-wavelength. To overcome this problem, we need to develop unwrapping methods for beyond the half-wavelength phase difference.

\section{References}

1) G. M. Nomarski, J. Phys. Radium Paris 16, 9S (1955).

2) M. Francon, Progress in Microscopy, Pergamon Press, p. 199 (1961).

3) T. Yatagai, Proc. SPIE, CR 46, 197 (1992).

4) R. S. Siroshi and G. S. Bhatnagar Opt. Acta 18 (1971), pp. 547-553.

5) H. Ishiwata, M. Itoh and T. Yatagai, Proc. SPIE, 2873 (1996), pp. 21-24. 
6) D. L. Lessor, J. S. Hartman and R. L. Gordon, J. Opt. Soc. Am., Vol. 69, No. 2 (1979), pp. 357-366.

7) J. S. Hartman, R. L. Gordon and D. L. Lessor, Appl. Opt., Vol. 19, No. 17 (1980), pp. 2998-3009.

8) J. S. Hartman, R. L. Gordon and D. L. Lessor, Appl. Opt., Vol. 20, No. 15 (1981), pp. 2665-2669.

9) M. R. Arnison, C. J. Cogswell, N. I. Smith, P. W. Fekete and K. G. Larkin, J. Microscopy, Vol. 199, Pt 1, July (2000), pp. 79-84.

10) M. Itoh, H. Ishiwata, A. Tsukada and T. Yatagai, Kôgaku (in Japanese), Vol. 30, No. 9 (2001), pp. 605-612.

11) E. B. van Munster, E. K. Winter, J. A. Aten, J. Microscopy, Vol. 191, Pt 2, August (1998), pp. 170-176.

12) H. Ishiwata, M. Itoh and T. Yatagai, Opt. Commun., Vol. 260, April (2006), pp. 117-126. 


\section{Figure captions}

Fig. 1 Components of light passed through a phase object with vector representation.

Fig. 2 Image components of a DIC image with vector representation.

Fig. 3 Block diagram of our equipment for surface metrology. A modulating retardation unit is built into the illuminating optics of a conventional microscope. Using a halogen lamp, it is possible to select the observation wavelength by varying the interference filter. We can control the retardation of the DIC microscope using the phase modulator. The phase modulator is controlled by a desktop computer to within $\pm 1^{\circ}$ accuracy. The CCD camera is a $1 / 3$ " (B/W) VGA type and is connected to a frame grabber in the desktop computer.

Fig. 4 Shape of the sample. The sample is a silicon wafer with grating patterns made using a semiconductor process. The grating patterns have line widths $\mathrm{L}$ ranging from $0.5 \mu \mathrm{m}$ to $10 \mu \mathrm{m}$ and are covered with an aluminum film to give phase objects with depth $\mathrm{d}$.

Fig. 5 DIC images of each region (a) to (c) taken by the $50 \times$ objective lens. (a): a photograph of region (a) including the line widths of 1.0, 1.2 and $1.4 \mu \mathrm{m}$; (b): region (b) including the line widths of 1.6 and $1.8 \mu \mathrm{m}$; (c): region (c) including the line widths of 2.0 and $3.0 \mu \mathrm{m}$.

Fig. 6 Measurement result by RM-DIC microscope based on the weak phase approximation. Horizontal axis is depth of sample. Vertical axis is measurement value.

Fig. 7 Measurement result of each region in the $75 \mathrm{~nm}$ sample assuming the phase approximation with cross-section.

(a): region (a) including the line widths of 1.0, 1.2 and $1.4 \mu \mathrm{m}$; (b): region (b) the line widths of 1.6 and $1.8 \mu \mathrm{m}$; (c): region (c) the line widths of 2.0 and $3.0 \mu \mathrm{m}$.

Fig. 8 Measurement results by SPC method and RZC method. The result by weak phase approximation is included for reference. Horizontal axis is depth of sample. Vertical axis is measurement value.

Table 1 Measurement value by weak phase approximation

Table 2 Measurement value by two-image SPC method

Table 3 Measurement value by RZC method

Table 4 Measurement value by three-image SPC method 


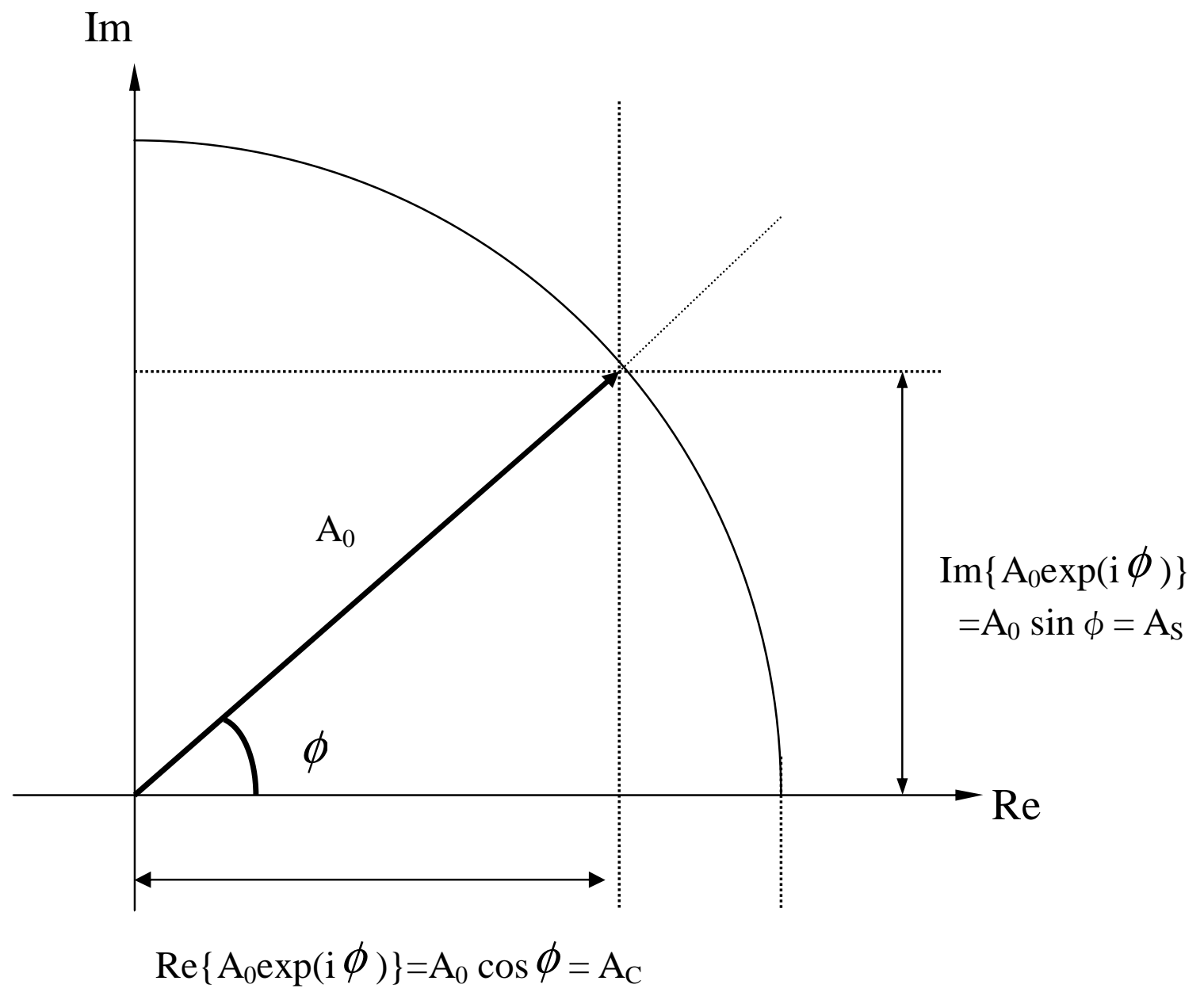

Fig.1 
Phase component

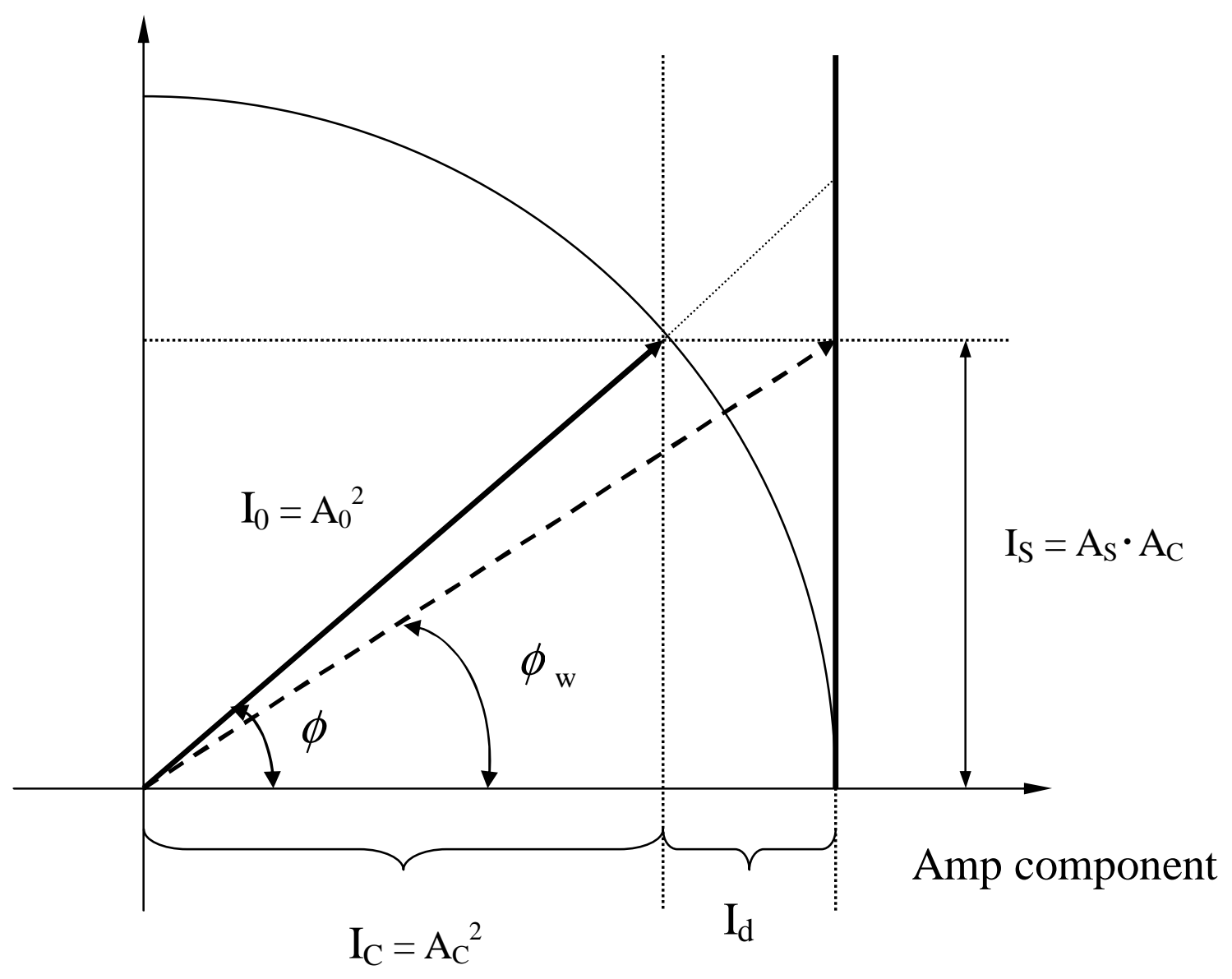

Fig.2 


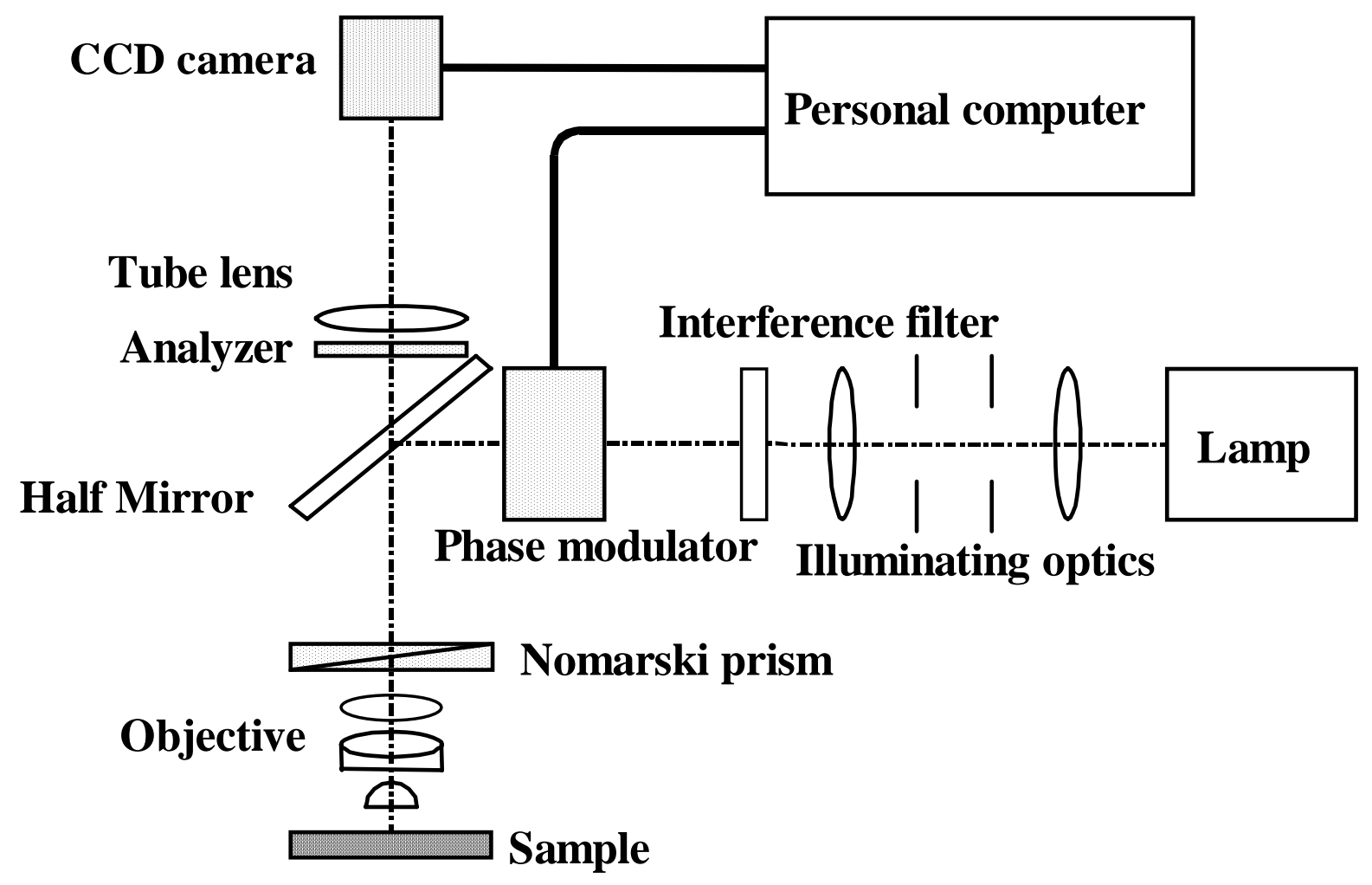

Fig.3 


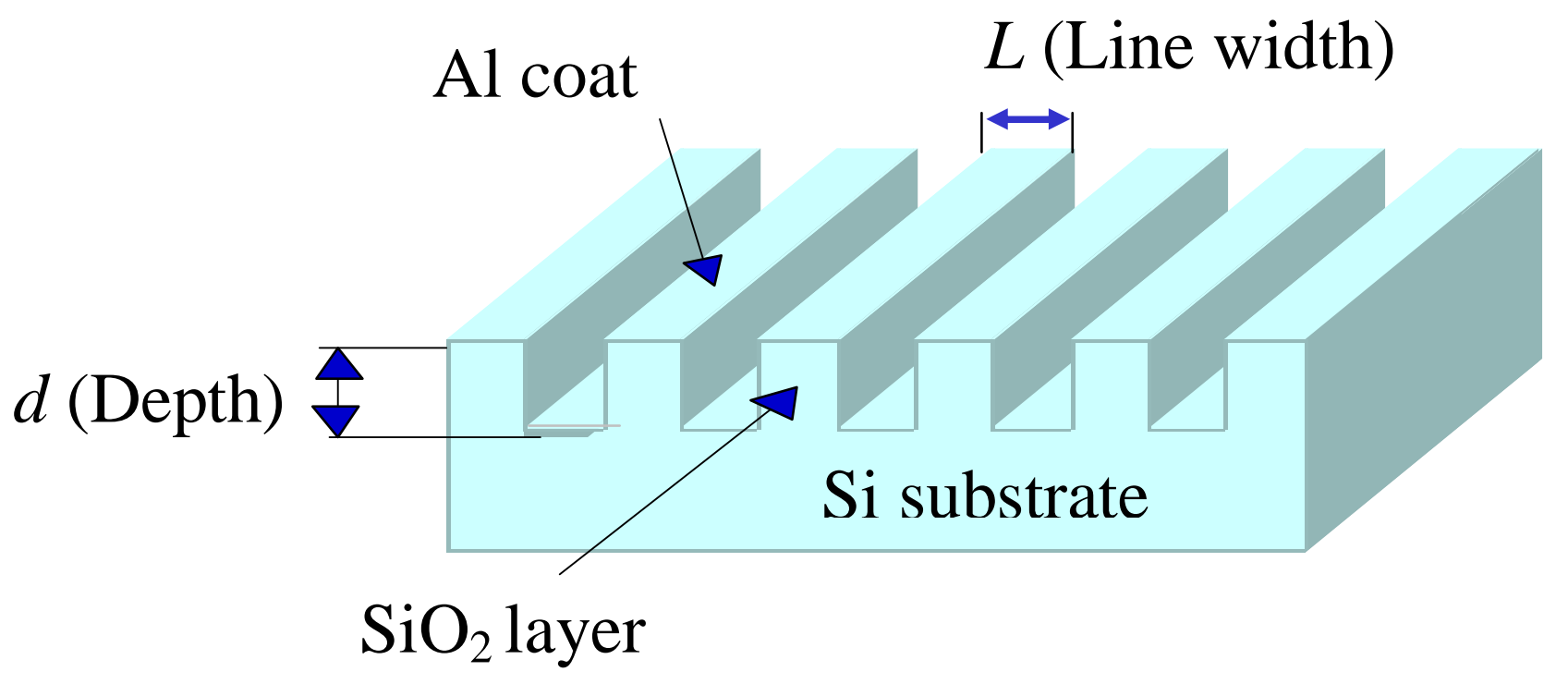

Fig. 4 


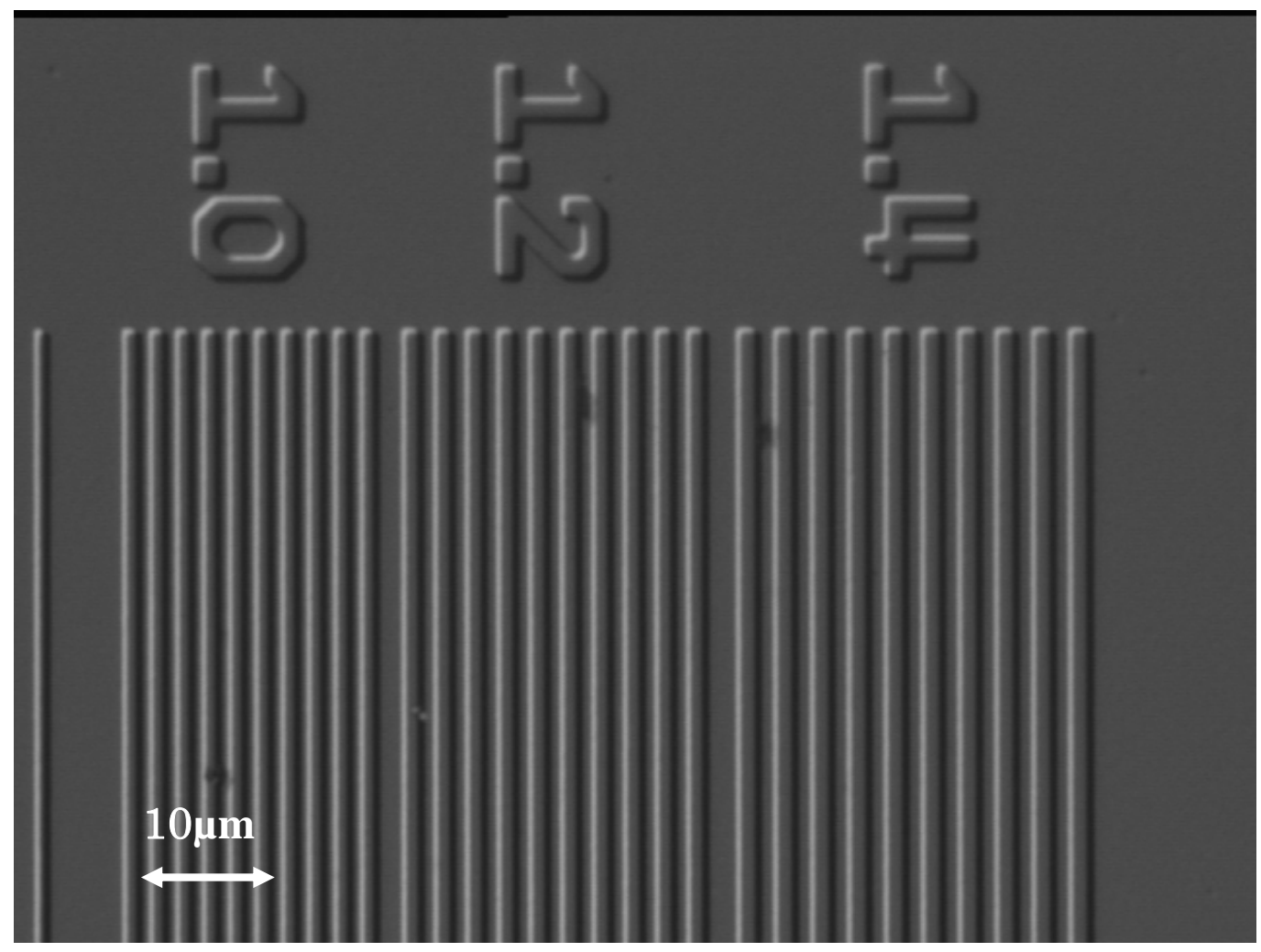

Fig.5 (a)

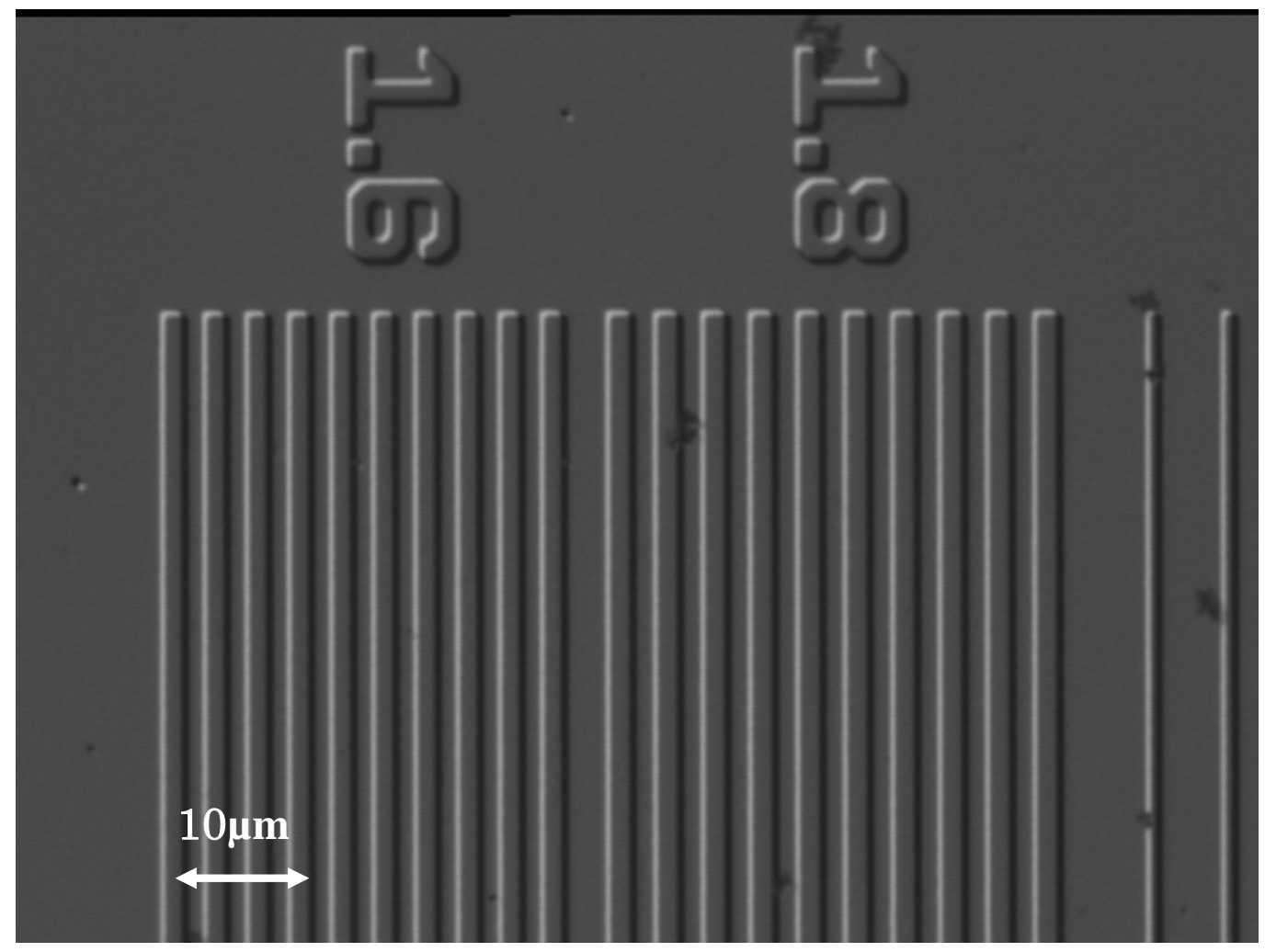

Fig.5 (b ) 


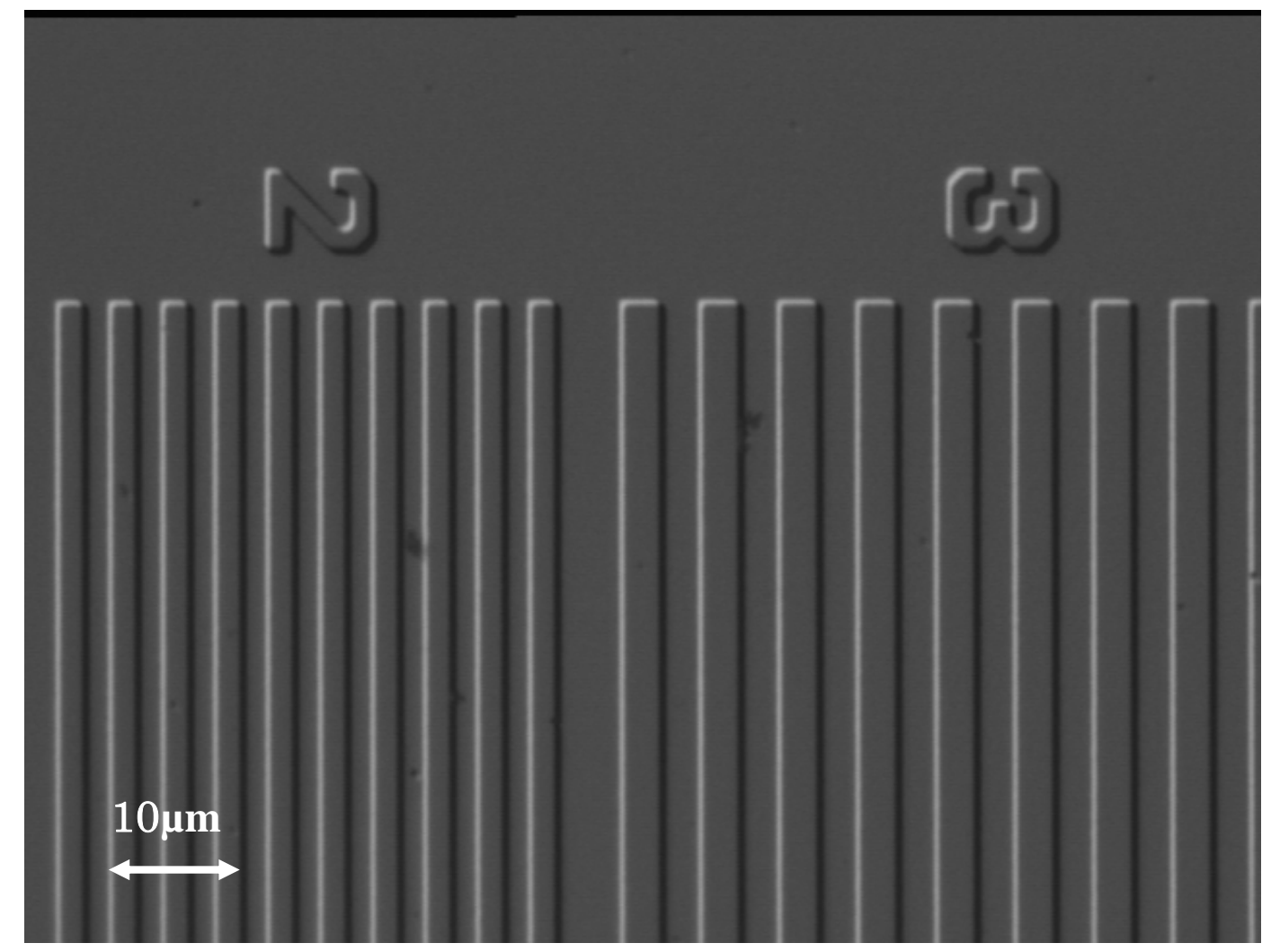

Fig.5 (c) 


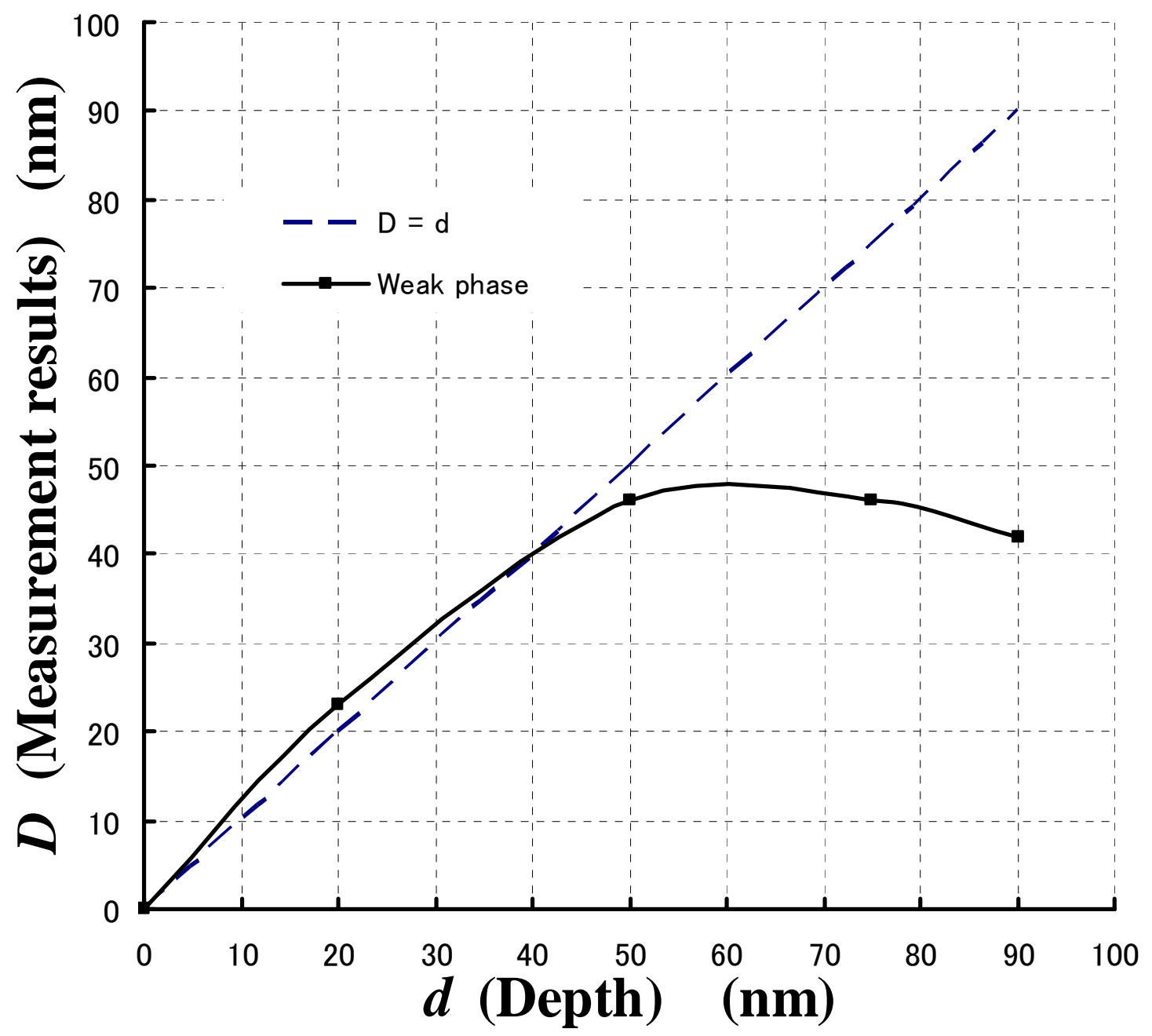

Fig.6 


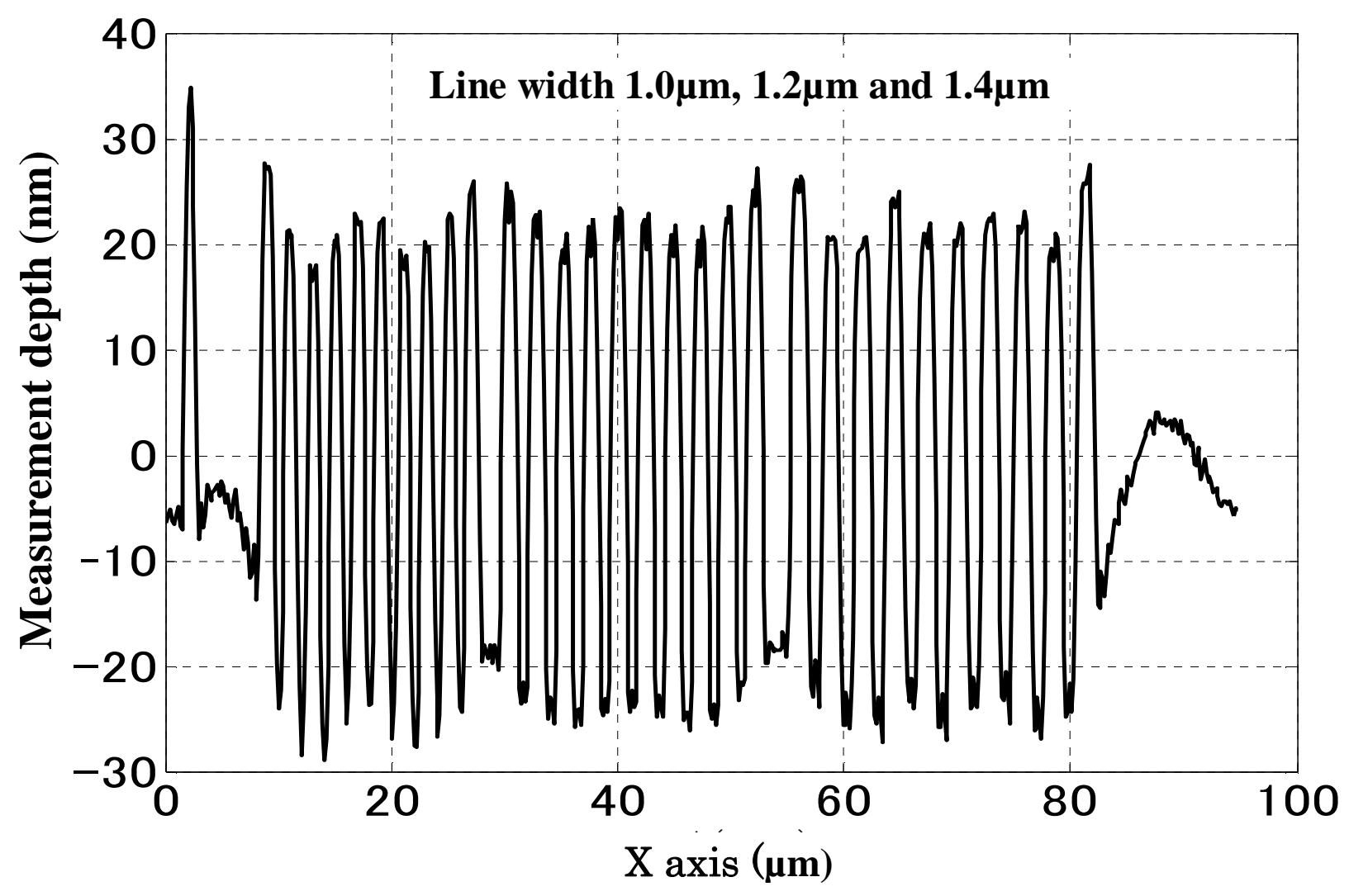

Fig. 7 (a)

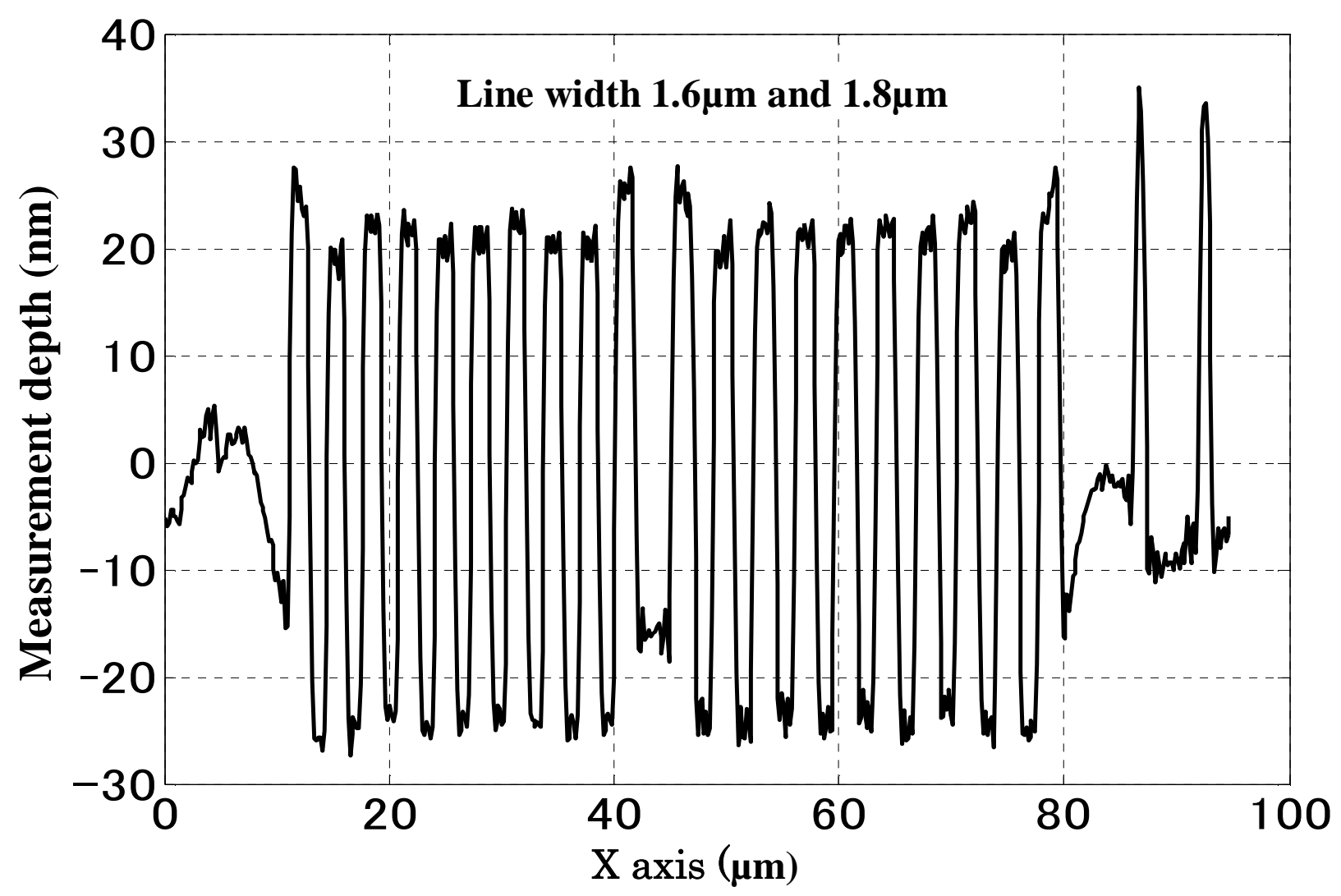

Fig. 7 (b) 


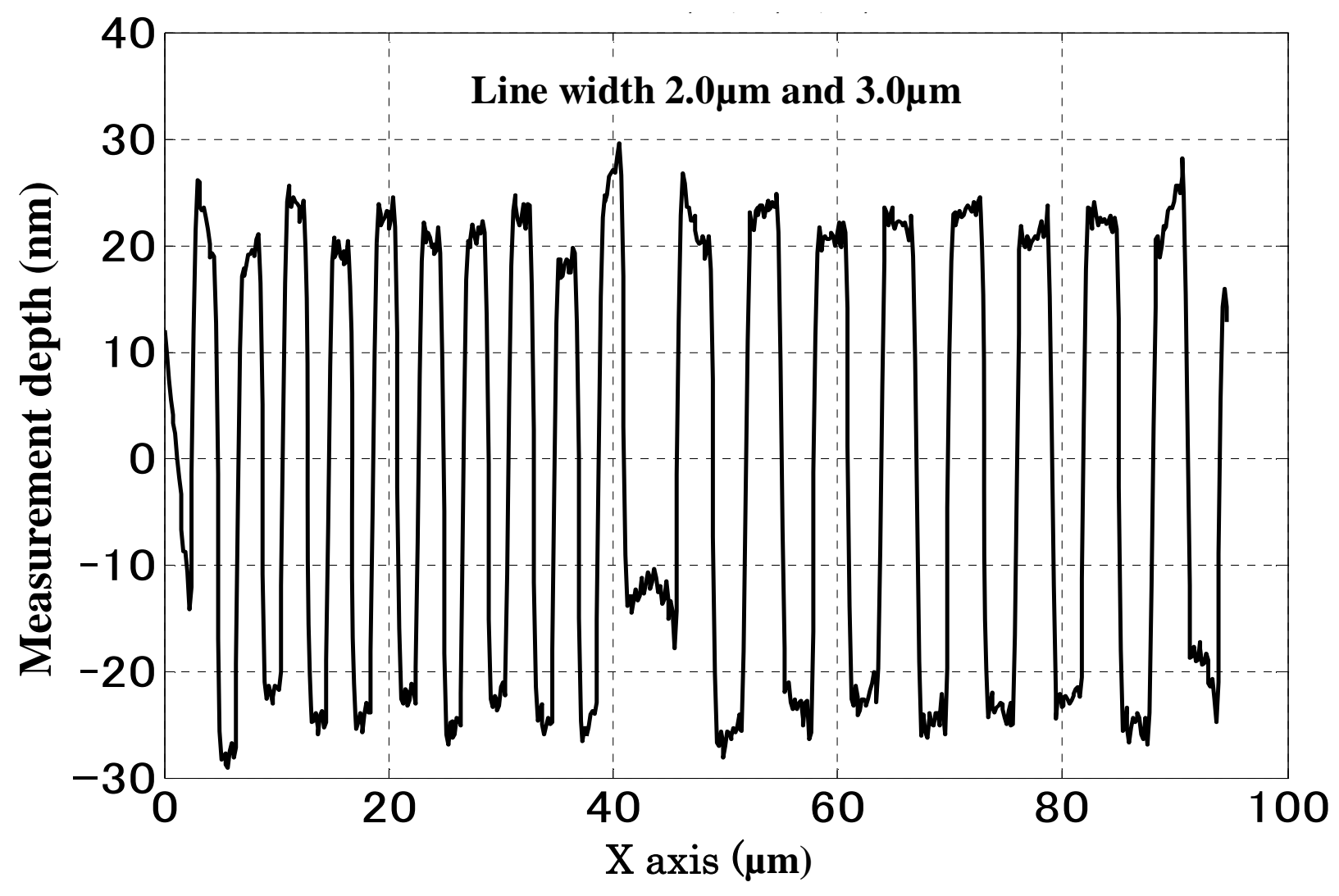

Fig. 7 (c) 


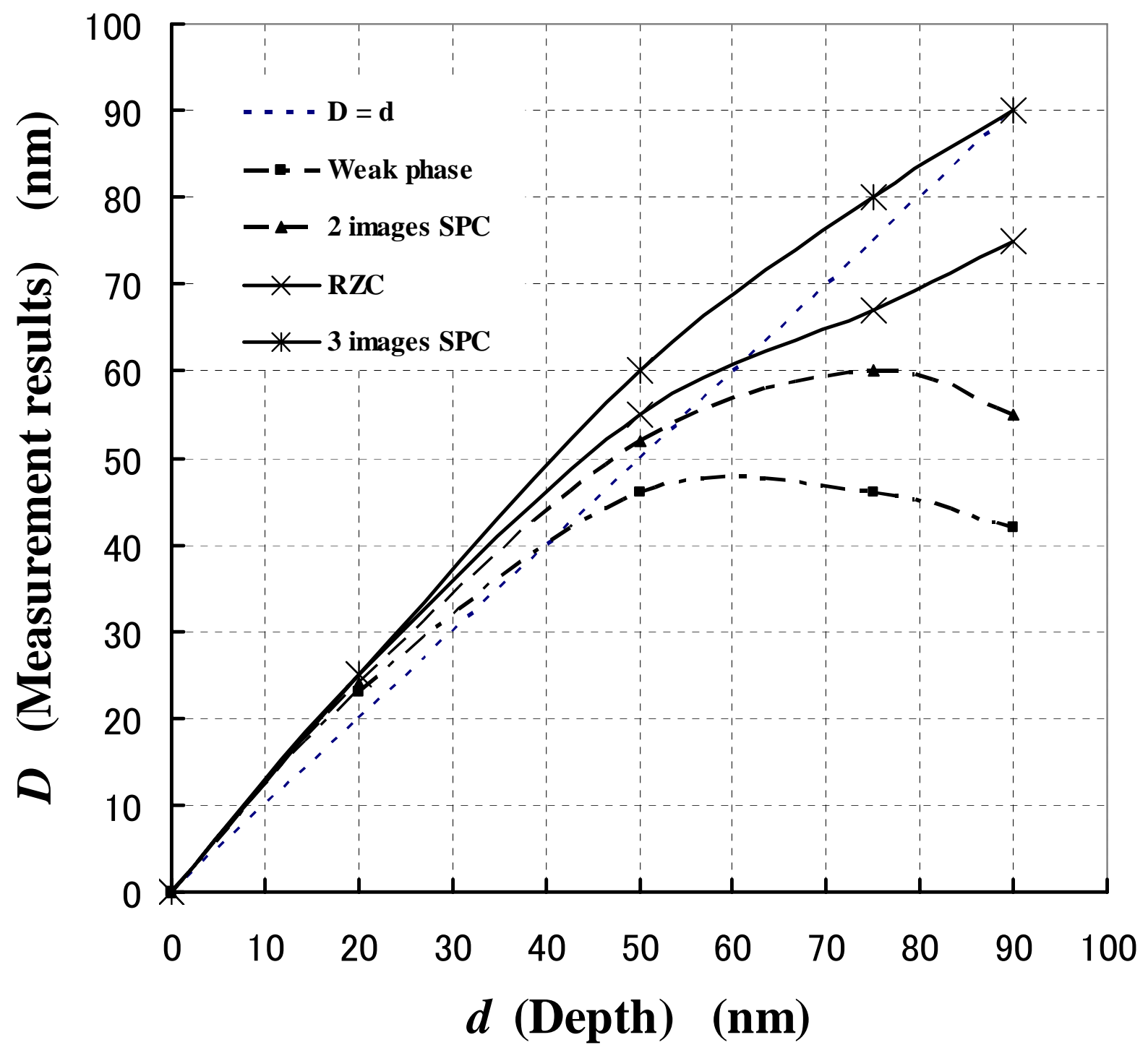

Fig.8 


\begin{tabular}{|c|c|c|c|c|c|c|c|c|}
\hline & \multicolumn{7}{|c|}{$D:$ Measurement results (nm) } \\
\hline & & \multicolumn{3}{|c|}{$\begin{array}{l}\text { Line width } \\
\text { ( region of a ) }\end{array}$} & \multicolumn{2}{|c|}{$\begin{array}{l}\text { Line width } \\
\text { ( region of } \mathbf{b} \text { ) }\end{array}$} & \multicolumn{2}{|c|}{$\begin{array}{l}\text { Line width } \\
\text { ( region of c ) }\end{array}$} \\
\hline & & $1.0 \mu \mathrm{m}$ & $1.2 \mu \mathrm{m}$ & $1.4 \mu \mathrm{m}$ & $1.6 \mu \mathrm{m}$ & $1.8 \mu \mathrm{m}$ & $2.0 \mu \mathrm{m}$ & $3.0 \mu \mathrm{m}$ \\
\hline \multirow{4}{*}{ 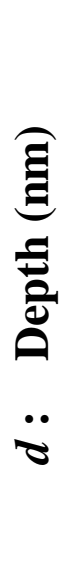 } & $25 \mathrm{~nm}$ & 23 & 23 & 23 & 23 & 23 & 23 & 23 \\
\hline & $50 \mathrm{~nm}$ & 46 & 46 & 46 & 46 & 45 & 45 & 45 \\
\hline & $75 \mathrm{~nm}$ & 48 & 45 & 45 & 45 & 44 & 48 & 46 \\
\hline & $90 \mathrm{~nm}$ & 44 & 43 & 43 & 43 & 43 & 42 & 42 \\
\hline
\end{tabular}

Table 1

\begin{tabular}{|c|c|c|c|c|c|c|c|c|}
\hline & \multicolumn{7}{|c|}{$D:$ Measurement results (nm) } \\
\hline & & \multicolumn{3}{|c|}{$\begin{array}{l}\text { Line width } \\
\text { ( region of a ) }\end{array}$} & \multicolumn{2}{|c|}{$\begin{array}{l}\text { Line width } \\
\text { ( region of } b \text { ) }\end{array}$} & \multicolumn{2}{|c|}{$\begin{array}{l}\text { Line width } \\
\text { ( region of c ) }\end{array}$} \\
\hline & & $1.0 \mu \mathrm{m}$ & $1.2 \mu \mathrm{m}$ & $1.4 \mu \mathrm{m}$ & $1.6 \mu \mathrm{m}$ & $1.8 \mu \mathrm{m}$ & $2.0 \mu \mathrm{m}$ & $3.0 \mu \mathrm{m}$ \\
\hline \multirow{4}{*}{ 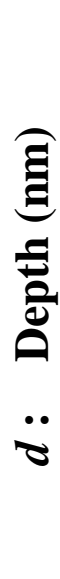 } & $25 \mathrm{~nm}$ & 24 & 24 & 24 & 24 & 24 & 23 & 23 \\
\hline & $50 \mathrm{~nm}$ & 52 & 52 & 52 & 52 & 52 & 50 & 50 \\
\hline & $75 \mathrm{~nm}$ & 60 & 60 & 60 & 60 & 60 & 60 & 60 \\
\hline & $90 \mathrm{~nm}$ & 54 & 55 & 55 & 55 & 55 & 55 & 55 \\
\hline
\end{tabular}




\begin{tabular}{|c|c|c|c|c|c|c|c|c|}
\hline & \multicolumn{7}{|c|}{$D:$ Measurement results (nm) } \\
\hline & & \multicolumn{3}{|c|}{$\begin{array}{l}\text { Line width } \\
\text { ( region a ) }\end{array}$} & \multicolumn{2}{|c|}{$\begin{array}{l}\text { Line width } \\
\text { ( region b ) }\end{array}$} & \multicolumn{2}{|c|}{$\begin{array}{l}\text { Line width } \\
\text { ( region c ) }\end{array}$} \\
\hline & & $1.0 \mu \mathrm{m}$ & $1.2 \mu \mathrm{m}$ & $1.4 \mu \mathrm{m}$ & $1.6 \mu \mathrm{m}$ & $1.8 \mu \mathrm{m}$ & $2.0 \mu \mathrm{m}$ & $3.0 \mu \mathrm{m}$ \\
\hline \multirow{4}{*}{ 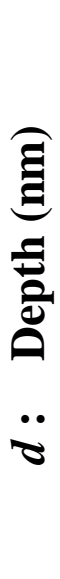 } & $25 \mathrm{~nm}$ & 23 & 23 & 23 & 26 & 26 & 25 & 26 \\
\hline & $50 \mathrm{~nm}$ & 55 & 54 & 55 & 55 & 55 & 56 & 56 \\
\hline & $75 \mathrm{~nm}$ & 68 & 67 & 67 & 65 & 66 & 66 & 66 \\
\hline & $90 \mathrm{~nm}$ & 77 & 77 & 75 & 76 & 75 & 73 & 73 \\
\hline
\end{tabular}

Table 3

\begin{tabular}{|c|c|c|c|c|c|c|c|c|}
\hline & \multicolumn{7}{|c|}{$D:$ Measurement results (nm) } \\
\hline & & \multicolumn{3}{|c|}{$\begin{array}{l}\text { Line width } \\
\text { ( region a ) }\end{array}$} & \multicolumn{2}{|c|}{$\begin{array}{l}\text { Line width } \\
\text { ( region b ) }\end{array}$} & \multicolumn{2}{|c|}{$\begin{array}{l}\text { Line width } \\
\text { ( region c ) }\end{array}$} \\
\hline & & $1.0 \mu \mathrm{m}$ & $1.2 \mu \mathrm{m}$ & $1.4 \mu \mathrm{m}$ & $1.6 \mu \mathrm{m}$ & $1.8 \mu \mathrm{m}$ & $2.0 \mu \mathrm{m}$ & $3.0 \mu \mathrm{m}$ \\
\hline \multirow{4}{*}{ 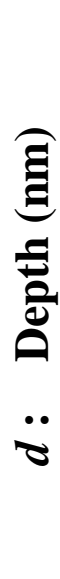 } & $25 \mathrm{~nm}$ & 25 & 25 & 26 & 26 & 26 & 25 & 25 \\
\hline & $50 \mathrm{~nm}$ & 63 & 60 & 60 & 60 & 60 & 60 & 60 \\
\hline & $75 \mathrm{~nm}$ & 80 & 78 & 78 & 83 & 83 & 80 & 83 \\
\hline & $90 \mathrm{~nm}$ & 92 & 92 & 90 & 90 & 90 & 88 & 88 \\
\hline
\end{tabular}

